\title{
LA ORGANIZACIÓN DE LAS NACIONES UNIDAS A LOS 75 AÑOS. CÓMO LOS RETOS DE HOY CONFIGURARÁN LOS SIGUIENTES 25 AÑOS*
}

\author{
THE UN AT 75. HOW TODAY'S CHALLENGES WILL \\ SHAPE THE NEXT 25 YEARS
}

\section{ANS DE L'ORGANISATION DES NATIONS UNIES. COMMENT LES DÉFIS ACTUELS DÉFINIRONT LES 25 PROCHAINES ANNÉES}

\author{
David M. Malone \\ Universidad de las Naciones Unidas, Japón \\ malone@unu.edu \\ Adam Day \\ Universidad de las Naciones Unidas, Japón \\ adam.day@unu.edu
}

Resumen: En el marco del 75 aniversario de la Organización de las Naciones Unidas (onU), celebrado en 2020, el artículo hace un balance de la situación y señala cómo la fuerza que ha perdido el multilateralismo en muchos de los temas más apremiantes a nivel global ha repercutido negativamente en varias de sus actividades fundamentales. La situación se evalúa en cuatro grandes campos de acción de las Naciones Unidas: paz y seguridad, desarrollo internacional, ayuda humanitaria y derechos humanos, y se argumenta que varios logros importantes alcanzados en cada rubro actualmente corren el riesgo de sufrir un retroceso o diluirse. En cada uno de estos grandes temas, se analizan los principales retos que enfrenta la organización, al tiempo que se proponen algunas líneas de acción que deberían profundizarse o hacia las que la onu debería reenfocar su labor.

La reflexión resulta más que pertinente dado que los retos que el mundo enfrenta hoy en día -particularmente los relacionados con el cambio climático,

* Se publica el artículo que aquí se ofrece por el acuerdo establecido entre Global Governance y Foro Internacional, según el cual ésta incluirá entre los propios un artículo de aquélla cada año y viceversa. 
pero también con los gigantescos cambios demográficos de las décadas por venir y el impacto de la tecnología- sólo pueden atenderse mediante la acción colectiva, y las Naciones Unidades deberían desempeñar un rol central al respecto.

Palabras clave: Naciones Unidas; ond; paz y seguridad; desarrollo internacional; ayuda humanitaria; derechos humanos,

ABSTRACT: Against the backdrop of the $75^{\text {th }}$ anniversary of the United Nations Organization (UN), celebrated in 2020, this paper takes stock of the situation and seeks to show how the strength lost by multilateralism in many of the most pressing issues on the global level has had a negative impact on several of the organization's fundamental activities. The situation is evaluated in four of the United Nation's major fields of activity: peace and security, international development, humanitarian aid and human rights. The paper argues that several of the significant advances made in each area now face the risk of setbacks or dilution. In each of these major issues, the main challenges facing the organization are analyzed, while proposing a number of lines of action that should be developed or towards which the un should reorient its work.

This review is of considerable relevance given the challenges the world faces today-particularly those related to climate change, but also the enormous demographic changes of the decades to come, and the impact of technologycan only be addressed through collective action, and the United Nations should play a central role in this regard.

Keywords: United Nations; un; peace and security; international development; humanitarian aid; human rights,

\section{Traducción de Fionn Petch, CM Idiomas}

RÉsumé: Dans le cadre du 75e anniversaire des Nations Unies (onU), célébré en 2020, l'article fait le point sur la situation et montre comment la force que le multilatéralisme a perdue dans plusieurs des problèmes les plus urgents au niveau mondial a eu un impact négatif sur plusieurs de ses activités principales. La situation est évaluée dans quatre principaux domaines d'action des Nations Unies: la paix et la sécurité, le développement international, l'aide humanitaire et les droits de l'homme, et on fait valoir que plusieurs réalisations importantes dans chaque domaine risquent actuellement d'être retardées ou diluées. Dans chacun de ces grands enjeux, sont analysés les principaux défis auxquels l'organisation est confrontée, tout en proposant des lignes d'ac- 
tion qui devraient être approfondies ou vers lesquelles l'onu devrait recentrer ses travaux.

La réflexion est plus que pertinente étant donné que les défis auxquels le monde est confronté aujourd'hui - en particulier ceux liés au changement climatique, mais aussi aux gigantesques changements démographiques des décennies à venir et à l'impact de la technologie - ne peuvent être relevés que par une action collective, et l'Organisation des Nations Unies doit jouer un rôle central à cet égard.

Mots clés: Nations Unies; onU; paix et sécurité; développement international; aide humanitaire; droits de l'homme.

Traducción de Rafael Segovia, CM Idiomas

Fecha de recepción: febrero de 2020

Fecha de aceptación: mayo de 2020

INTRODUCGIÓN

$\mathrm{E}$ ste año, la Organización de las Naciones Unidas (ONU) cumple 75 años. Como muchos septuagenarios, la Organización puede parecer un poco alejada de la realidad, quizás algo sorda y, de manera evidente, con menor capacidad para reunir a grandes potencias en torno a una meta común. De hecho, muchas de las tendencias actuales parecerían presionar a la onU hacia una irrelevancia cada vez mayor: muchas de las fuerzas que, en los últimos treinta años, impulsaron la actuación de las Naciones Unidas se han ido alejando progresivamente de la organización. Las tensiones Este-Oeste aumentan, y no hay indicios de que la onU esté proporcionando un foro que promueva su desescalada. Incluso tres miembros permanentes (3P) del Consejo de Seguridad han dado muestras de desacuerdo en contextos como el de Yemen, Libia y el mandato de las operaciones de paz. Para exacerbar lo anterior, las agendas nacionalistas alrededor del mundo reducen las probabilidades de cooperación internacional y abren un espacio para que los actores 
regionales adopten posturas más firmes. No deja de ser revelador que este año, el Secretario General de las Naciones Unidas, António Guterres, hablara de la "crisis" en las contribuciones a la organización, lo que pudiera leerse como una advertencia de un repliegue mucho más tangible. ${ }^{1}$

En este artículo, evaluamos la situación en cuatro grandes campos de acción de las Naciones Unidas: paz y seguridad, desarrollo internacional, ayuda humanitaria y derechos humanos. Sostenemos que hay logros importantes en cada rubro que, hoy por hoy, corren el riesgo de sufrir un retroceso o diluirse. Dentro del radio de acción sustantiva de la organización, los enfoques multilaterales están perdiendo relevancia en las tareas de disminución del riesgo de conflicto, la construcción de un desarrollo sostenible, la satisfacción de las necesidades de las poblaciones vulnerables y el apoyo para establecer sociedades incluyentes que rindan cuentas, además de que hay un creciente número de líderes nacionales que consideran que algunos -o la mayoría- de estos objetivos son superfluos o nocivos para sus propios intereses cada vez más estrechos. Este escenario resulta particularmente preocupante si pensamos que los desafíos más importantes del mundo actual no pueden enfrentarse con iniciativas de un solo Estado: la cooperación multilateral es el único camino eficaz. Ésa fue la cruda enseñanza de las terribles consecuencias de las guerras napoleónicas del siglo xix y de las dos guerras mundiales del siglo pasado: cada una de ellas desencadenó esfuerzos multilaterales.

\section{PAZ Y SEGURIDAD}

A lo largo de su historia, el Consejo de Seguridad de las Naciones Unidas ha transitado por altas y bajas, pero el periodo

${ }^{1}$ António Guterres, Palabras pronunciadas ante el Quinto Comité de la Organización de las Naciones Unidas: "Improving the Financial Situation of the Organization", Nueva York, 4 de junio de 2019. 
inmediato posterior a la Guerra Fría fue en el que desplegó mayor actividad y cobró particular relevancia. Tras su audaz actuación ante la invasión iraquí a Kuwait en 1990, el Consejo mostró una unidad nunca antes vista: en unos cuantos años inició 15 nuevas operaciones de mantenimiento de la paz y adoptó 200 resoluciones. ${ }^{2}$ Sin embargo, desde la icónica unidad de propósito internacional que suscitaron los acontecimientos del 11 de septiembre, el Consejo se ha caracterizado, en muchos aspectos, más por la ruptura y la parálisis que por la acción, como fue evidente principalmente con la invasión de Estados Unidos a Iraq en 2003, aunque también después de la intervención de 2011 en Libia. ${ }^{3} \mathrm{Si}$ bien hubo algunas áreas importantes de cooperación en ese lapso -como la creación de operaciones de mantenimiento de la paz y de misiones políticas especiales, y la adopción de nuevos regímenes de sanciones continuaron a pasos acelerados-, las fisuras en el interior del Consejo se han profundizado en los últimos años. Actualmente, el antagonismo entre China, Rusia y Estados Unidos parece estar revirtiendo la postura del Consejo de Seguridad a una postura de Guerra Fría, lo que potencialmente podría debilitar espacios clave de cooperación global, incluyendo la no proliferación, la lucha contra el terrorismo y el mantenimiento de la paz. Por su parte, los líderes de la Unión Europea difieren de los tres países arriba mencionados cada vez con mayor frecuencia. ${ }^{4}$

En cuanto a su objetivo primordial -poner fin a las guerras-, el Consejo de Seguridad parece ser, en aspectos funda-

${ }^{2}$ Mats Berdal, "The Security Council and Peacekeeping", en Vaughan Lowe, Adam Roberts, Jennifer Welsh y Dominik Zaum (comps.), The United Nations Security Council and War: The Evolution of Thought and Practice since 1945 (pp. 174-205), Oxford, University Press, 2008, p. 176.

${ }^{3}$ Sebastian Einsiedel, David M. Malone y Bruno Stagno Ugarte (comps.), The Security Council in the 21st Century, Boulder, Lynne Rienner, 2015.

${ }^{4}$ Richard Gowan, "Minimum Order: The Role of the Security Council in an Era of Major Power Competition", Nueva York, Centro de Investigación sobre Políticas de la Universidad de las Naciones Unidas, marzo de 2019. 
mentales, cada vez más impotente. Ha fracasado en atajar fuertes guerras en Siria y Yemen, a pesar del masivo sufrimiento humano y de una clara amenaza a la paz y seguridad que trasciende las fronteras de esos dos países. Obstaculizado por la falta de unidad de los cinco miembros permanentes (5P), el Consejo no ha podido actuar en Ucrania, se ha mantenido al margen ante la gradual descomposición de Venezuela y se le margina cuando se trata de evitar la crisis entre Estados Unidos e Irán. En ámbitos en los que el Consejo ya ha actuado, la evidencia señala que las Naciones Unidas tienen ahora menor capacidad que en otras épocas para contener y gestionar conflictos violentos: las dinámicas de conflicto en Malí, Libia, Sudán y Sudán del Sur parecen no verse mayormente afectadas ante la considerable presencia de misiones de la onU en sus territorios. Más aún, hay personal de mantenimiento de la paz destacado en países en los que se observan menos de $10 \%$ de muertes relacionadas con un conflicto lo que, en una escala global, aumenta la creciente sensación de irrelevancia. ${ }^{5}$

En este contexto, algunos expertos han hecho referencia a la "crisis de legitimidad" del Consejo. ${ }^{6}$ Otros han señalado que su capacidad para desempeñar el papel de gobernanza global previsto en la Carta languidece a pesar de la necesidad exponencial de una respuesta multilateral a las amenazas actuales. ${ }^{7}$ De hecho, el Consejo no es siquiera una referencia cuando se necesita hacer frente a algunos de los riesgos de seguridad emergentes más importantes, como los que representan las cibertecnologías y la inteligencia arti-

${ }^{5}$ Bruce Jones, Charles T. Call, Daniel Touboulets y Jason Fritz, Managing the New Threat Landscape: Adapting the Tools of International Peace and Security, Washington, D.c., The Brookings Institution, septiembre de 2017.

${ }^{6}$ Ian Martin, "In Hindsight: What's Wrong with the Security Council?”, Security Council Report, 29 de marzo de 2018.

7 David Bosco, "Assessing the UN Security Council: A Concert Perspective”, Global Governance 20, 2014, p. 12. 
ficial. ${ }^{8}$ Más bien, hoy en día, gran parte del discurso en las Naciones Unidas versa sobre las reducciones en el gasto presupuestario dedicado al pilar de la paz y seguridad que, en los últimos años, se ha reducido de 8000 a menos de 7000 millones de dólares. ${ }^{9}$ El presupuesto regular de la organización ha ido disminuyendo, particularmente en términos reales (ajustado a la inflación), debido, en parte, a su propia planificación, pero también a una morosidad creciente en el pago de cuotas o a la inhabilidad de algunos países de cubrirlas. Estas tendencias ofrecen a las grandes potencias un número menor de puntos de consenso, menores oportunidades de colaborar frente a las amenazas que van surgiendo y, por lo tanto, un mayor riesgo potencial de ser ellos quienes entren en conflicto. ${ }^{10}$

Existen signos preocupantes que indican que, en las tres tareas sustantivas del Consejo de Seguridad, la fuerza y la unidad de las Naciones Unidas están todavía más en riesgo. El retiro estadounidense, en 2018, del acuerdo nuclear avalado por la onU con Irán y del tratado de armas nucleares de alcance intermedio es señal de que la confianza en los enfoques multilaterales relacionados con las armas de destrucción masiva está disminuyendo. ${ }^{11} \mathrm{El}$ aumento en la crítica a la forma en que los $5 \mathrm{P}$ podrían estar instrumentalizando a las Naciones Unidas a favor de acciones de mano dura en la lucha contra el terrorismo ${ }^{12}$ y las fricciones sobre las respues-

${ }^{8}$ Eleonore Pauwels, "The New Geopolitics of Converging Risks: The UN and Prevention in an Era of Al", Nueva York, Centro de Investigación sobre Políticas de la Universidad de las Naciones Unidas, 2019.

${ }^{9}$ Naciones Unidas, Asamblea General, Fifth Committee Meeting, UN Doc. GA/AB/4319 (6 de mayo de 2019).

${ }^{10}$ Berdal, art. cit., p. 179 .

${ }^{11}$ Moritz Pieper, "An Iran Nuclear Deal without the United States? Chinese, European, and Russian Interests and Options after the US Withdrawal from the Joint Comprehensive Plan of Action", en Fu-Lai Tony Yu y Diana S. Kwan (comps.), Contemporary lssues in International Political Economy, Singapur, Palgrave Macmillan, 2019, pp. 35-53.

${ }^{12}$ Sebastian Einsiedel y David M. Malone, "Security Council", en Thomas G. Weiss y Sam Daws, (comps.), The Oxford Handbook on the United 
tas contra el terrorismo en Sahel podrían ser un presagio del mermado papel que desempeñará la organización en el futuro. En cuanto al mantenimiento de la paz, el probable esfuerzo del gobierno estadounidense actual por reducir aún más el presupuesto, aunado a las divisiones en el proceso de retirada de las grandes misiones en Darfur y la República Democrática del Congo, podría significar que el alcance de la cooperación entre los $5 \mathrm{P}$ también se está contrayendo. ${ }^{13}$

Ante estas circunstancias, quizá sea prudente reenfocar la energía hacia las cuestiones en que las Naciones Unidas siguen siendo claramente relevantes para la paz y seguridad internacionales y hacia donde el Consejo de Seguridad ha mostrado un interés de colaboración común en la no proliferación, la lucha contra el terrorismo y algunas operaciones de mantenimiento de la paz. Un ejemplo clave es el caso de la República Popular Democrática de Corea: en medio de grandes divisiones entre Estados Unidos, Rusia y China, el Consejo fue capaz de seguir fortaleciendo las sanciones de la ONU, lo que subraya el potencial de la colaboración y la singularidad del foro que ofrece la Organización cuando se logra el acuerdo de las tres potencias militares más prominentes de nuestro tiempo. ${ }^{14} \mathrm{El}$ consenso del Consejo sobre la destrucción de las armas químicas de Siria en 2013 reforzó el argumento de que la onu sigue siendo una piedra angular de los compromisos mundiales contra la proliferación. ${ }^{15}$ Del mismo modo, en el campo de la lucha contra el terrorismo,

Nations (2 ${ }^{\text {da }}$ ed.), Oxford, University Press, 2018, pp. 140-165; Jane E. Stromseth, "An Imperial Security Council? Implementing Security Council Resolutions 1373 and 1390", American Society of International Law Procedure, 97, 2003, p. 43.

13 Fred Carver, "Peacekeeping Budget Approval and Cuts Leave Fundamental Questions Unaddressed”, International Peace Institute Global Observatory, 14 de septiembre de 2018.

${ }_{14}$ Naciones Unidas, Consejo de Seguridad, Security Council, Resolución 2397. S/ RES/2397, 22 de diciembre de 2017. Gowan, op. cit.

15 Richard Price, "Syria and the Chemical Weapons Taboo", Journal of Global Security Studies 4 (1), 2019, pp. 37-52. 
el Consejo y la Asamblea General adoptaron resoluciones ambiciosas y de gran alcance en el periodo subsiguiente al 11 de septiembre para hacer frente tanto a combatientes extranjeros como al apoyo a grupos e individuos terroristas. ${ }^{16}$ Finalmente, los esfuerzos de mantenimiento de la paz en África continúan siendo un tema destacado en el que, a pesar de las divisiones, las principales potencias han alcanzado unanimidad. Combinados, el destacamento de una nutrida misión de mantenimiento de la paz en Malí y el acuerdo generalizado de la trayectoria de las misiones de la onu en la República Centroafricana, Sudán del Sur y la República Democrática del Congo, son muestra de que sí puede haber concierto dentro del Consejo de Seguridad.

Viendo hacia el futuro, es probable que surjan amenazas nuevas o se intensifiquen algunas ya existentes, y que se conviertan en el centro de la arquitectura de las Naciones Unidas en el terreno de la paz y la seguridad. Lo anterior incluye la forma en que el cambio climático incide en los riesgos de seguridad, la manera en que las nuevas tecnologías podrían converger para plantear peligros inéditos y el acelerado impacto del cambio demográfico y la urbanización. ${ }^{17}$ Es crucial que las principales potencias encuentren mecanismos de colaboración en torno a estos retos globales en el entendido de que será costoso enfrentarlos. Sin embargo, resulta igualmente importante que recordemos la amenaza existencial que representan las armas de destrucción masiva y el papel absolutamente indispensable que la onU puede desempeñar para contenerla y reducirla. Mantener el consenso en este eje podría requerir esfuerzos en otras esferas, como el avance en la construcción de confianza en el ámbito de la lucha contra el terrorismo, el mantenimiento de la paz o, de hecho, en los demás pilares de la organización, mismos que se analizan más adelante.

${ }^{16}$ Sebastian Einsiedel, “Assessing the UN's Efforts to Counter Terrorism”, 2016 Global Terrorism Index, Institute for Economics and Peace, Sidney, diciembre de 2016.

17 Pauwels, art. cit. 


\section{Desarrollo internacional}

Desde un inicio, la onU fue concebida para no ser un jugador importante en el campo del desarrollo internacional. Fundada un año después de que las instituciones de Bretton Woods -el Fondo Monetario Internacional (FMI) y el Banco Mundial (Bм) - se desarrollaran en 1944 como las herramientas principales para evitar que volviera a levantarse la ola proteccionista de la década de 1920, el diseño de las Naciones Unidas obedeció a la determinación de Franklin D. Roosevelt y Winston Churchill de impedir que se repitieran los errores del Tratado de Versalles de 1919, que había sido excesivamente punitivo con las potencias derrotadas en la Primera Guerra Mundial. ${ }^{18}$ Por su parte, el objetivo del FMI era fomentar una administración económica sólida en el interior de los países miembros y contribuir así a una economía global sana y a una estabilidad financiera global. El BM, en cambio, se centró inicialmente en la reconstrucción de las naciones devastadas como consecuencia de la Segunda Guerra Mundial.

La falta de ambiciones en temas económicos y sociales es evidente en la Carta de las Naciones Unidas, particularmente en relación con las considerables facultades que se le otorgan para responder ante amenazas a la paz. El capítulo IX de la Carta apenas contiene una breve referencia a la "cooperación económica y social internacional" y anticipa la coordinación de "distintos organismos especializados [...] en materias de carácter económico, social, cultural, educativo, sanitario y otras conexas". En la configuración que la Carta da al Consejo Económico y Social (Ecosoc) -diseñado para "iniciar estudios e informes (...) y emitir recomendaciones"se vislumbra el descuido relativo en el que, durante décadas,

${ }^{18}$ La frustrada Organización Internacional de Comercio, concebida por los aliados de los tiempos de guerra, fue -en cierta medida- resarcida con la creación, en 1948, del Acuerdo General sobre Aranceles Aduaneros y Comercio (GATT), que a su vez fue sustituido, en 1993, por un organismo más ambicioso y universal: la Organización Mundial del Comercio. 
estaría el trabajo de la organización en el campo económico. De hecho, el repetido fracaso que desde su creación ha tenido el Ecosoc por destacar, a pesar de los múltiples intentos por reformarlo e inyectar voluntad política a sus decisiones, refleja, por un lado, la preferencia de los países industrializados de hacer caso omiso de la onU cada vez que hay grandes sumas de dinero en juego y, por el otro, la impotencia de los países en vías de desarrollo para desafiar efectivamente esa tendencia. En resumen, ha sido patente que las acciones a favor del desarrollo hayan ido a parar, cada vez con mayor frecuencia, a donde están los recursos: en las instituciones de Bretton Woods, con sede en Washington; en los bancos regionales de desarrollo y en otras instituciones que no están bajo la jurisdicción de la Asamblea General.

A lo largo de la década de 1950, la descolonización se aceleró, lo que transformó profundamente el papel de las Naciones Unidas en materia de desarrollo: en este rubro, la agenda de los países recién independizados obtuvo cada vez mayor relevancia, restando así atención a la reconstrucción de posguerra. Fue entonces que el rápido crecimiento de una faceta de desarrollo en las agencias especializadas de la ONU se convirtió en el vector más eficaz en las tareas de desarrollo, centrado principalmente en las necesidades de los países en vías de desarrollo. El tímido trabajo inicial en el Sur Global -a menudo realizado bajo el nombre de "cooperación técnica”- pronto se transformó en equipos importantes de la onu que buscaban apuntalar el desarrollo de principio a fin. A las agencias de las Naciones Unidas que, en términos generales, todavía mantienen una sólida reputación de efectividad, entre otras cosas por su impacto en la protección y promoción del desarrollo -como el Fondo de las Naciones Unidas para la Infancia (Unicef), la Organización Mundial de la Salud (oms) y el Programa Mundial de Alimentos (WFP, por sus siglas en inglés) - se unieron otras agencias, fondos y programas de la Organización con reputaciones más variadas (en el mejor de los casos), muchas de las cuales, con demasiada frecuencia, se han visto asociadas 
con una mala administración y corrupción (su operación, en la mayoría de los casos, se basa primordialmente en prácticas clientelares, un fenómeno más difícil de erradicar que la malversación). El frecuentemente innovador Programa de las Naciones Unidas para el Desarrollo (PNUD), que defendió con éxito el concepto de "desarrollo humano" en la década de 1990, nunca logró atraer el nivel de apoyo de patrocinadores que hubiera necesitado para alcanzar la influencia comparativa de las instituciones financieras internacionales (IFI), que continuaron siendo el canal de financiamiento a los países en vías de desarrollo preferido por los países industrializados. ${ }^{19}$ El breve intento por favorecer el desarrollo redistributivo a través del Nuevo Orden Económico Mundial contribuyó a esta tendencia.

Por lo tanto, si bien la onU llegó a ser considerada un jugador significativo en la configuración de la agenda normativa para el desarrollo a nivel mundial, la organización brilló mucho menos en el papel de implementador de programas para el desarrollo. Lo anterior fue particularmente cierto durante las crisis de deuda que se sucedieron en el decenio de 1980, cuando las IFI impusieron a los países en vías de desarrollo excesivamente endeudados las ahora desacreditadas políticas de "ajuste estructural" a las que siguieron, de forma más generosa, la radical reducción o cancelación de deuda de la década de 1990, cuyos términos se negociaron en un espacio distinto. ${ }^{20}$ Las acciones programáticas de las IFI se llevaron a cabo mayoritariamente fuera del ámbito de las Naciones Unidas. Sin embargo, el papel de la organización como defensor del desarrollo creció con un nivel relativa-

${ }^{19}$ En el campo del desarrollo, y más aún en el de los esfuerzos y la asistencia humanitaria, la oNU ha establecido frecuentemente alianzas con organizaciones no gubernamentales nacionales e internacionales y generalmente ha obtenido resultados extraordinarios. Por sus mandatos y cultura, no es raro que las instituciones financieras internacionales vean este enfoque con cierto recelo.

${ }^{20}$ En el llamado Club de París para países acreedores y en el Club de Londres para instituciones comerciales acreedoras. 
mente menor de cuestionamientos en los años posteriores a la Guerra Fría, cuando el Banco Mundial, el Fondo Monetario Internacional y la Organización Mundial del Comercio empezaron a cooperar de forma más cercana en los esfuerzos de la onU (aunque hubo agencias, como el Unicef, que mantuvieron su postura crítica sobre los programas de ajuste estructural del Banco). No fue hasta finales del decenio de 1990 que los países en vías de desarrollo realmente reafirmaron su postura a través de las Naciones Unidas, lo que dio como resultado el establecimiento de los Objetivos de Desarrollo del Milenio (oDM) de 2001. Formados por ocho objetivos simples y admirablemente claros, 21 metas específicas y 61 indicadores, los oDM eran medibles y fáciles de comprender. Y mejor aún, tras una ola sostenida de crecimiento en África y Asia durante los primeros quince años del siglo XxI y de políticas sociales creativas en América Latina en el mismo periodo, se avanzó muy significativamente en alcanzar los objetivos planteados. Este resultado condujo a que, en las capitales nacionales del mundo y en las Naciones Unidas, surgiera la convicción de que podría lograrse una serie de objetivos más ambiciosos, lo que, a su vez, dio origen a los Objetivos de Desarrollo Sostenible (oDs). Impulsados por la visión de contener y revertir la degradación ambiental, reducir los riesgos de conflicto y construir instituciones incluyentes, entre otros, los oDs son mucho más demandantes que los oDM, aunque su mayor nivel de integración podría resultar útil durante la implementación. Sin embargo, es posible que los oDs sean excesivamente ambiciosos para países con recursos limitados, situación que se combina con un crecimiento económico mundial desacelerado, con mayores tensiones entre las principales potencias y con la consecuente marginalización de las preocupaciones de muchas regiones en vías de desarrollo.

Entonces, ¿dónde estamos ahora?

$\mathrm{Al}$ momento de escribir estas líneas, muchos gobiernos consideran que el cambio climático es el desafío global abrumador, y que es costoso enfrentarlo. Sin embargo, la retira- 
da del gobierno estadounidense durante la administración del presidente Donald Trump del Tratado de París de 2016 socavó la solidaridad internacional para atender el tema de manera urgente y brindar el financiamiento que el programa requiere. Para 2019, muchas economías emergentes con influencia (la India, Brasil) contribuían poco para obtener resultados medioambientales positivos en su carrera por alcanzar un crecimiento económico, aunque éste haya empeorado la degradación medioambiental en cada uno de sus países. En el otro lado de la moneda está China que, a pesar de haberse distinguido por apalancar nuevas tecnologías lucrativas de energía limpia, sigue contaminando en exceso.

La dificultad de administrar la implementación de los oDs -que contemplan 179 metas y mucho más de 200 indicadores-, el riesgo de un nuevo aumento global de deuda por los países en vías de desarrollo y la imposibilidad de predecir las maniobras geoestratégicas de las tres potencias principales y de muchos otros líderes nacionalistas y autoritarios menores en cada uno de los continentes se conjuntan para crear una interrogante grave sobre el panorama del ritmo con el que se pudiera alcanzar un mayor desarrollo económico. Los catastróficos incendios de 2019 en el Amazonas, que excedieron a los anteriores en intensidad y daños, y los huracanes que acosaron al Caribe en tiempos recientes, ocasionando más daños que antes, son un recordatorio de que las condiciones creadas por el hombre interactúan con la naturaleza generando desastres que, con frecuencia, tienen un ímpetu terrible, y que hay líderes nacionales obsesionados consigo mismos que, con demasiada frecuencia, son esquivos o elusivos mientras la temperatura del mundo aumenta y los más pobres de sus habitantes sufren inmensamente.

La asistencia para el desarrollo sigue siendo primordial, particularmente para sociedades y economías frágiles que, por lo general, intentan recuperarse de situaciones de guerra. Sin embargo, en términos reales, el apoyo disminuye con lentitud y gran parte del financiamiento que se asigna bajo el rubro de "ayuda" no se canaliza para alcanzar un desarrollo a 
largo o mediano plazo, sino para aliviar la aflicción humanitaria intensa y políticamente cinética a corto plazo. Cada vez más, los programas humanitarios están destinados a atender el naufragio en el que las luchas políticas internas (con distintos niveles de interferencia del exterior) dejan a sus países. Así ha sucedido en Siria, Yemen, Sudán del Sur, Somalia y la región del Sahel. Por otro lado, no es raro que la asistencia para el desarrollo sirva para subsidiar préstamos comerciales otorgados con fines de desarrollo, lo que contribuye al endeudamiento general en lugar de brindar un subsidio. Además, quienes dominan la mayor parte del desarrollo fundamental son actores bilaterales (las inversiones chinas en África son un buen ejemplo), mientras la ONU parece consumirse atendiendo una crisis presupuestal tras otra.

\section{Asistencia HUMANitARia}

En los últimos 75 años, el impacto más visible y medible de la ONU a nivel mundial quizá esté en el frente humanitario, gracias al auge de agencias de prominencia mundial, como Fondo de las Naciones Unidas para la Infancia (UNICEF), el Alto Comisionado de las Naciones Unidas para los Refugiados (ACNUR), la Organización de las Naciones Unidas para la Alimentación y la Agricultura (FAO) y el World Food Program (WFP). Más de 60 millones de refugiados que escapaban de la persecución, la violencia y la guerra han recibido ayuda de la ONU y, cada año, alrededor de 80 millones de personas que viven en condiciones extremadamente vulnerables reciben una dieta básica. ${ }^{21}$ Desde 1946, las Naciones Unidas ha salvado la vida de más de 90 millones de niños, lo

${ }^{21}$ Naciones Unidas, Global Humanitarian Overview 2019, Oficina de la Coordinación de Asuntos Humanitarios, https://www.unocha.org/publication/global-humanitarian-overview/global-humanitarian-overview-2019 
que le mereció el Premio Nobel de la Paz. ${ }^{22}$ La capacidad de la organización de entregar ayuda humanitaria expedita y eficazmente se ha incrementado notablemente a lo largo de sus 75 años de vida. Por ejemplo, la creación en 2005 del Fondo Central para la Acción en Casos de Emergencia (cERF, por sus siglas en inglés) permitió multiplicar el volumen y la velocidad de la respuesta humanitaria y, desde entonces, la ONU ha gastado más de 5000 millones de dólares para el alivio humanitario en más de 100 países. ${ }^{23}$

A pesar de que el financiamiento humanitario ha aumentado exponencialmente en las últimas dos décadas, hoy en día, las tendencias humanitarias muestran una brecha cada vez más grande entre lo que puede ofrecer la onu y las necesidades de las poblaciones vulnerables. En este momento hay más desplazados como consecuencia de conflictos que casi en cualquier otra época desde la Segunda Guerra Mundial, lo que contribuye al actual récord histórico de personas que sufren inseguridad alimenticia por esa causa. ${ }^{24}$ Hoy día, las crisis humanitarias no sólo afectan a más seres humanos, sino que su duración es mayor: una crisis promedio persiste durante más de nueve años y exige la mayor parte de los recursos humanitarios de la organización. A lo largo del último lustro, cuatro de estas crisis perennes -Somalia, Sudán del Sur, Sudán y Siria- ocuparon más de la mitad del financiamiento total bajo el rubro de respuesta humanitaria. ${ }^{25}$ En Yemen, con 24 millones de individuos que necesitan protección y asistencia humanitaria, se vive la mayor crisis humani-

22 Julia Zorthian, "5 United Nations Achievements Worth Celebrating”, Time, 23 de octubre de 2015, https://time.com/4085757/unitednations-achievements/

${ }^{23}$ Naciones Unidas, Central Emergency Response Fund (sitio de internet), 2019, https://cerf.un.org

${ }^{24}$ Naciones Unidas, Alto Comisionado para los Refugiados, "Global Trends: Forced Displacement in 2017”, 2017, https://www.unhcr.org/ globaltrends2017/

${ }^{25}$ Naciones Unidas, Global Humanitarian Overview 2019, Oficina de la Coordinación de Asuntos Humanitarios, https://www.unocha.org/publi cation/global-humanitarian-overview/global-humanitarian-overview-2019 
taria en el mundo, y la situación sigue empeorando. Un denominador común en estos casos es la falta de un prospecto inmediato de solución que resuelva el aspecto político subyacente en los conflictos. Las potencias regionales e internacionales han mostrado, particularmente en Siria y Yemen, una intención más marcada de luchar por sus propias agendas regionales que de ofrecer resoluciones políticas sostenibles, lo que afianza la dinámica del conflicto y reduce el margen de maniobra de las Naciones Unidas para facilitar el fin de las hostilidades.

Si bien los contextos de conflicto representan las peores amenazas en la agenda humanitaria, los riesgos relacionados con el clima y los desastres naturales también van en aumento. El año pasado, los desastres naturales afectaron a 350 millones de personas, causaron daños por miles de millones de dólares y ejercieron aún más presión sobre la ya de por sí débil capacidad de respuesta de muchos países vulnerables. ${ }^{26}$ El cambio climático tiene severos efectos negativos en las cosechas, el agua y el sustento de las personas. Las secuelas más duras las sufren países con altos niveles de pobreza, países que atraviesan por un conflicto o aquellos que sufren ambos males. ${ }^{27}$ En sitios con añejas necesidades humanitarias -como Afganistán o Sudán del Sur- cada vez hay mayor evidencia de que el cambio climático recrudece el riesgo humanitario para grandes conjuntos poblacionales. ${ }^{28}$ En la región del Sahel, el cambio climático está a punto de generar algunos de los riesgos transversales más agudos a nivel mundial debido a la enorme dependencia que existe de la agricultura de secano (dependiente de la lluvia), el rápido crecimiento poblacional, la vulnerabilidad ante la sequía, la inestabilidad política y las débiles capacidades del

${ }^{26}$ Naciones Unidas, Secretario General, Annual Report on the Work of the Organisation, UN Doc. A/73/1 (2018), https://www.un.org/annualreport/2018/

${ }^{27}$ Cruz Roja de Noruega, "Overlapping Vulnerabilities: The lmpacts of Climate Change on Humanitarian Needs", Cruz Roja de Noruega, 2019.

${ }^{28}$ Loc. cit. 
Estado para satisfacer los requerimientos cotidianos de sus ciudadanos. $^{29}$

Viendo a futuro, es sumamente probable que las necesidades humanitarias sigan creciendo al mismo ritmo que ahora, o incluso más rápido, y que sobrepasen con un margen todavía mayor la capacidad de respuesta humanitaria de la ONU y otras organizaciones. Este año presenta un crudo panorama del rumbo que estamos siguiendo: alrededor de $150 \mathrm{mi}-$ llones de personas necesitan asistencia humanitaria, lo que implica una suma superior a 26000 millones de dólares. Sin embargo, será imposible atender a alrededor de $40 \%$ de la población necesitada, y los niveles de financiamiento no han alcanzado el $35 \%$ de lo que hace falta para cubrir las exigencias actuales. Los conflictos violentos -causa primordial de las crisis humanitarias- no parecen disminuir, y es muy probable que alimenten la tendencia ya existente hacia un atrincheramiento más profundo y de mayor riesgo para la sociedad civil. ${ }^{30}$ La aparición de nuevas amenazas, como el resurgimiento del ébola en el este del Congo y las crisis políticas de Burkina Faso y Camerún, seguramente incrementarán aún más el costo y la complejidad de la respuesta humanitaria.

La dirección tomada apunta hacia un desafío fundamental del sistema humanitario: es indispensable brindar ayuda de emergencia inmediata capaz de salvar vidas, pero ésta suele suministrarse en un contexto de crisis prolongadas que duran una década o más. Si bien se trata de un trabajo que evidentemente alivia una enorme cantidad de sufrimiento humano, no aporta necesariamente el tipo de capacidades locales y nacionales que permitirían a los países que experimentan el conflicto satisfacer, a más largo plazo, los requerimientos de las comunidades y tener un desarrollo económico y social más amplio. Iniciativas clave, como Nueva Forma de Trabajar y Buenas Prácticas en Materia de Donaciones Huma-

${ }^{29}$ US Agency for International Development, "Climate Change Risk Profile: The Sahel”, 13 de abril de 2017.

${ }^{30}$ Einsiedel et al., art. cit. 2017. 
nitarias, podrían conducir a respuestas más coordinadas ante la emergencia y a modalidades de financiamiento más predecibles. Sin embargo, todavía no se establece el tejido conectivo entre la provisión de ayuda humanitaria y el tipo de desarrollo sustentable que se necesita para proteger de crisis recurrentes a los países en riesgo. ${ }^{31}$

Aunque el análisis anterior sobre la polarización entre las principales potencias y el creciente nacionalismo pudiera ser indicio de una parálisis frente a los riesgos, existen, por lo menos, algunos signos esperanzadores de que el sistema multilateral se está adaptando, más o menos, a las causas interrelacionadas que avivan las crisis humanitarias. En 2018, la mayoría de los Estados Miembros -con la notable excepción, en ambos casos, de Estados Unidos- avalaron sendos pactos globales sobre migración y refugiados, en evidente reconocimiento de la necesidad de colaborar para proteger a algunas de las poblaciones más vulnerables del mundo. La Agenda 2030 para el Desarrollo Sostenible también vincula claramente los objetivos globales generales con los que hacen frente específicamente a los riesgos humanitarios. Aunque éstas sean señales políticas útiles, por sí solas resultan insuficientes para vencer los desafíos que plantean las tendencias actuales.

\section{DeRechos Humanos}

A lo largo de los últimos setenta años, las Naciones Unidas ha encabezado un cambio transformacional en aspectos normativos, legales y pragmáticos de los derechos humanos. La Declaración Universal de 1948 se ha ampliado significativamente y hay tratados que detallan los derechos económicos, sociales, civiles y políticos. ${ }^{32}$ Ahora, la protección explícita

${ }^{31}$ Good Humanitarian Donorship (sitio de internet), 2019, https:// www.ghdinitiative.org/ghd/gns/home-page.html

${ }^{32}$ Simon Chesterman, David M. Malone y Santiago Villalpando, "Introduction", en The Oxford Handbook of UN Treaties, Oxford, University Press, 2019, pp. 1-23. 
de mujeres y niños se acepta casi universalmente, y los derechos de personas lesbianas, gays, bisexuales, transgénero y queer (LGBTQ) mejoran globalmente (aunque no de manera uniforme y con algunos retrocesos) ${ }^{33}$ Existe un consenso generalizado en el principio de que las violaciones graves a los derechos humanos no deben quedar sin castigo, y muchas de las principales normas internacionales en la materia han sido incorporadas en las legislaciones nacionales a nivel mundial. ${ }^{34}$ Tras el surgimiento y caída de la Comisión de Derechos Humanos, el Consejo de Derechos Humanos desempeña hoy en día un papel evidente en la evaluación de los expedientes de derechos humanos de todos los países y cuenta con plataformas optimizadas para la defensa y rendición de cuentas que le permiten actuar con más fuerza y mayor alcance. ${ }^{35}$ De hecho, la aprobación de los ops subraya a qué grado los derechos humanos forman ya parte del discurso internacional sobre desarrollo y prevención de la violencia. Los oDs hacen referencia al derecho humano al agua y a la alimentación, a la salud y a una vida en paz.

Sin embargo, en muchos aspectos, la agenda de las Naciones Unidas en el terreno de los derechos humanos parece estar deteriorándose, y muchos de los avances por los que se había luchado arduamente corren el riesgo de sufrir un revés. Tres de los cinco miembros permanentes del Consejo de Seguridad tienen reticencias para reconocer la aplicabilidad universal de las normas de derechos humanos en temas como las políticas y prácticas migratorias, la libertad religio-

33 Naciones Unidas, Asamblea General, Convention on the Elimination of All Forms of Discrimination Against Women, A/HRC/RES/32/2, 18 de diciembre de 1979, United Nations Treaty Series, vol. 1249, 18 de diciembre de 1979.

34 Naciones Unidas, Asamblea General, Resolución A/RES/73/12, 22 de diciembre de 2018, Asamblea General de las Naciones Unidas, Rome Statute of the International Criminal Court, 17 de julio de 1998.

35 Naciones Unidas, Consejo de Derechos Humanos, "The Protection and Promotion of Human Rights in the Philippines", UN Doc. A/ HRC/41/L.20, 7 de mayo de 2019. 
sa, las detenciones masivas y el rígido acotamiento del espacio político. En respuesta, la tendencia de la onU ha sido favorecer la "diplomacia discreta" en todos los casos de violaciones a los derechos humanos, salvo en los más flagrantes, con la intención de proteger el acceso de todos los países a estos derechos. Lo anterior le ha ganado la crítica severa de los defensores más preeminentes. ${ }^{36}$ Muy bien, hacer caso omiso de la crítica puede funcionar, pero sólo hasta cierto punto: si no quiere echar por la borda su credibilidad, que le es vital, la organización tendrá que tomar una decisión respecto a este tipo de temas de derechos humanos básicos. Se han puesto a prueba distintos enfoques para abrir un camino a través de la maleza del nacionalismo y de la exacerbada alergia interna a la crítica internacional. Zeid Ra'ad, el valiente Alto Comisionado de las Naciones Unidas inmediato anterior, era un antagonista equitativo de todos los gobiernos con violaciones a los derechos humanos que, si bien recibía en privado el aplauso de muchos colegas dentro del sistema de las Naciones Unidas, sólo contaba abiertamente con el apoyo de un número reducido de Estados. La nueva Alta Comisionada, antes presidenta de Chile y altamente respetada, empezó a trazar un mapa distinto desde las primeras etapas de su gestión: se está enfocando más en enfrentar transversalmente los retos en materia de derechos humanos en un momento de la historia en que las grandes potencias no los valoran y el populismo en los ámbitos nacionales podría estar ejerciendo una presión contraria sobre los avances más importantes. ${ }^{37} \mathrm{El}$ retiro de Estados Unidos del Consejo de Derechos Humanos fue otro signo negativo.

La manera inadecuada en que la onu responde actualmente a algunas de las situaciones de derechos humanos más atroces evidencia su desasosiego. La parálisis de la organiza-

36 Kenneth Roth, "Why the UN Chief's Silence on Human Rights Is Deeply Troubling", The New York Times, 24 de abril de 2019.

${ }^{37}$ Human Rights Watch, "Report on the United States", 2019, https:/ / www.hrw.org/world-report/2019/country-chapters/united-states 
ción ante la tortura y la matanza sistemáticas de cientos de miles de musulmanes rohingyas en Myanmar es tan sólo un ejemplo. Su inhabilidad para evitar los asesinatos a gran escala en Yemen y Siria también ponen de relieve el limitado impacto de los esfuerzos tradicionales encabezados por Estados miembros. La detención china de alrededor de un millón de musulmanes uigur en campos de "reeducación" no ha provocado ninguna respuesta seria de las Naciones Unidas. La falta de respuestas verosímiles ante el homicidio de Yamal Jhashoggi, el uso impune de armas químicas del régimen sirio y la revocación de la visa estadounidense a la fiscal general de la Corte Penal Internacional se suman a los inquietantes indicadores de que, hoy por hoy, los gobiernos nacionales que oponen resistencia o violan las normas e instituciones de derechos humanos no enfrentan mayores repercusiones.

Las tendencias en el terreno de los derechos humanos son preocupantes. Una encuesta de 2018 encontró un aumento vertiginoso del autoritarismo a nivel global así como un severo desapego respecto a las obligaciones jurídicas internacionales en muchas partes del mundo. ${ }^{38}$ Lo anterior podría estar contribuyendo a otras predisposiciones negativas, incluyendo las que tienen que ver con la no discriminación, la libertad de expresión y los derechos de los trabajadores. ${ }^{39}$ De hecho, a menos que el rumbo actual se corrija en forma decidida, existe un riesgo real de que los enormes logros de los últimos 70 años o más se vean erosionados y rechazados o, incluso, que se reviertan. Más aún, el financiamiento para algunos de los componentes más importantes de la maquinaria crítica de los derechos humanos podría sufrir recortes en los próximos años, lo que dejaría a la organización atada de pies y manos para la siguiente crisis. Aunque el nombramiento de Michelle Bachelet como Alta Comisionada es esperanzador,

${ }^{38}$ World Justice Project, “Rule of Law Index: 2017-2018”, 2018, https:/ / worldjusticeproject.org/our-work/research-and-data/wjp-rule-law-index2017\%E2\% $80 \% 932018$

${ }^{39}$ Loc. cit. 
su firme liderazgo no es suficiente para vencer la inercia y el abuso de derecho en los Estados miembros a nivel global.

\section{Conclusiones}

Hemos pintado un cuadro lúgubre de gran parte de las funciones sustantivas de las Naciones Unidas. En realidad, el hecho de que el multilateralismo esté perdiendo fuerza en muchos de los temas más apremiantes a nivel global justo cuando en 2020 se celebró el 75 aniversario de la onU, representa un verdadero reto para la credibilidad de la organización que, además, parece estar a la defensiva en muchos de los aspectos más cruciales de su labor. Lo anterior, a pesar del grado en que las últimas siete décadas y media se fueron configurando positivamente gracias a acuerdos multilaterales, muchos de los cuales habían mantenido su fuerza y utilidad hasta hace poco. Los niveles sin precedente de cooperación internacional en materia económica, social y política, a los que apuntala una gama impresionante de instituciones multilaterales, son el resultado directo de muchos de los logros más importantes del mundo desde 1945, y así debe reconocerse. Las Naciones Unidas siguen siendo el principal foro universal para discutir el marco normativo que sostiene el desarrollo global, y las agencias del sistema de la onU en distintas partes del mundo siguen participando en un trabajo programático en apoyo directo de los oDs.

Sin embargo, la creciente anarquía entre potencias prominentes ha significado que el papel y la credibilidad de casi todos los actores multilaterales se hayan visto afectados considerablemente. Lo que el secretario general Guterres ha llamado "déficit de confianza" en el orden multilateral actual es una advertencia; hasta ahora la brecha no sólo se mantiene, sino que cada vez es más amplia. ${ }^{40}$

${ }^{40}$ Naciones Unidas, Secretario General, Annual Report on the Work of the Organisation, UNDoc. A/73/1 (2018), https:/ /www.un.org/annualreport/2018/ 
Los enfoques multilaterales que han mantenido las armas de destrucción masiva bajo un control considerable, que han forjado un progreso histórico en los objetivos de desarrollo, que han contribuido a las normas universales de derechos humanos y generado una respuesta humanitaria a gran escala, corren el riesgo de que se les pase por alto o de sufrir un revés. A los retos que el mundo enfrenta en la actualidad -particularmente los relacionados con el cambio climático, pero también con las gigantescas transformaciones demográficas de las décadas por venir y el impacto de la tecnologíasólo se les puede atender a través de una acción colectiva. Si bien este artículo se terminó de escribir antes de que estallara la pandemia de Covid-19, esta crisis mundial no hace más que subrayar las cuestiones expuestas con anterioridad. Aunque la propuesta del Secretario General para un cese al fuego universal a fin de mitigar el sufrimiento humano haya sido ampliamente aplaudida, languideció en el Consejo de Seguridad que, por su parte, no ha logrado tomar ninguna medida significativa en este trance. De hecho, muchas de las principales potencias -y de manera más evidente Estados Unidos- han mostrado enorme desinterés en la cooperación internacional para contener el virus; más bien han optado por intercambiar acusaciones. En este contexto, el peligro de que veamos un retroceso mayor en el éxito que las Naciones Unidas ha alcanzado en materia de paz y seguridad, el desarrollo, la asistencia humanitaria y los derechos humanos es ahora todavía más grande. No obstante, la necesidad de cooperación multilateral nunca ha sido tan importante como en este momento. La onu puede ayudar en todos y cada uno de estos desafíos, pero... ¿`será bienvenida su colaboración?

Traducción de Gonzalo Celorio, GM Idiomas 


\section{BibLIOGRAFÍA}

Berdal, Mats, "The Security Council and Peacekeeping”, en Vaughan Lowe, Adam Roberts, Jennifer Welsh y Dominik Zaum (comps.), The United Nations Security Council and War: The Evolution of Thought and Practice since 1945 (pp. 174-205), Oxford, University Press, 2008.

Binder, Martín y Monica Heupel, "The Legitimacy of the UN Security Council: Evidence from Recent General Assembly Debates”, International Studies Quarterly 59 (2), 2015, pp. 238-250.

Bosco, David, "Assessing the UN Security Council: A Concert Perspective”, Global Governance 20, 2014, pp. 545-562.

Carver, Fred, "Peacekeeping Budget Approval and Cuts Leave Fundamental Questions Unaddressed”, International Peace lnstitute Global Observatory, 14 de septiembre de 2018.

Chesterman, Simon, David M. Malone y Santiago Villalpando, "Introduction", en The Oxford Handbook of UN Treaties, Oxford, University Press, 2019, pp. 1-23.

Cruz Roja de Noruega, "Overlapping Vulnerabilities: The lmpacts of Climate Change on Humanitarian Needs", Cruz Roja de Noruega, 2019.

Einsiedel, Sebastian, "Assessing the UN's Efforts to Counter Terrorism”, 2016 Global Terrorism Index, Institute for Economics and Peace, Sidney, diciembre de 2016.

Einsiedel, Sebastian y David M. Malone, "Security Council", en Thomas G. Weiss y Sam Daws, (comps.), The Oxford Handbook on the United Nations (2da ed.), Oxford, University Press, 2018, pp. 140-165.

Einsiedel, Sebastian, David M. Malone y Bruno Stagno Ugarte (comps.), The Security Council in the 21st Century, Boulder, Lynne Rienner, 2015.

Einsiedel, Sebastian, Louise Bosetti, Cale Salih, Wilfred Wan y James Cockayne, "Civil War Trends and the Changing Nature of Armed Conflict”, Nueva York, Centro de Investigación sobre Políticas de la Universidad de las Naciones Unidas, 2017.

Good Humanitarian Donorship (sitio de internet), 2019, https:// www.ghdinitiative.org/ghd/gns/home-page.html 
Gowan, Richard, "Minimum Order: The Role of the Security Council in an Era of Major Power Competition", Nueva York, Centro de Investigación sobre Políticas de la Universidad de las Naciones Unidas, marzo de 2019.

Guterres, António, Palabras pronunciadas ante el Quinto Comité de la Organización de las Naciones Unidas: "Improving the Financial Situation of the Organization", Nueva York, 4 de junio de 2019.

Human Rights Watch, "Report on the United States”, 2019, https:/ / www.hrw.org/world-report/2019/country-chapters/unitedstates

Jones, Bruce, Charles T. Call, Daniel Touboulets y Jason Fritz, Managing the New Threat Landscape: Adapting the Tools of International Peace and Security, Washington, D.c., The Brookings Institution, septiembre de 2017.

MARTIN, Ian, "In Hindsight: What's Wrong with the Security Council?”, Security Council Report, 29 de marzo de 2018.

Naciones Unidas, Asamblea General, Convention on the Elimination of All Forms of Discrimination Against Women, A/HRC/ RES/32/2, 18 de diciembre de 1979, United Nations Treaty Series, vol. 1249, 18 de diciembre de 1979.

Naciones Unidas, Asamblea General, Resolución A/RES/73/12, 22 de diciembre de 2018, Asamblea General de las Naciones Unidas, Rome Statute of the International Criminal Court, 17 de julio de 1998

Naciones Unidas, Asamblea General, Resolución de la Asamblea General A/RES/70/1, 21 de octubre de 2015.

Naciones Unidas, Alto Comisionado para los Refugiados, "Global Trends: Forced Displacement in 2017”, 2017, https://www.un hcr.org/globaltrends2017/

Naciones Unidas, Consejo de Seguridad, Security Council, Resolución 2397. S/ RES/2397, 22 de diciembre de 2017.

Naciones Unidas, Secretario General, Annual Report on the Work of the Organisation, UN Doc. A/73/1 (2018), https://www.un. org/annualreport/2018/

Naciones Unidas, "70 Ways the UN Makes a Difference”, 2019, https://www.un.org/un70/en/content/70ways/index.html 
Naciones Unidas, Asamblea General, Fifth Committee Meeting, UN Doc. GA/AB/4319, 6 de mayo de 2019.

Naciones Unidas, Central Emergency Response Fund (sitio de internet), 2019, https://cerf.un.org

Naciones Unidas, Consejo de Derechos Humanos, "The Protection and Promotion of Human Rights in the Philippines", UN Doc. A/HRC/41/L.20, 7 de mayo de 2019.

Naciones Unidas, Global Humanitarian Overview 2019, Oficina de la Coordinación de Asuntos Humanitarios, https://www.unocha.org/publication/global-humanitarian-overview/globalhumanitarian-overview-2019

Naciones Unidas, Secretario General, "Message on the International Day of Multilateralism and Diplomacy for Peace", 24 de abril de 2019.

Pauwels, Eleonore, "The New Geopolitics of Converging Risks: The UN and Prevention in an Era of Al", Nueva York, Centro de Investigación sobre Políticas de la Universidad de las Naciones Unidas, 2019.

Pieper, Moritz, "An Iran Nuclear Deal without the United States? Chinese, European, and Russian Interests and Options after the US Withdrawal from the Joint Comprehensive Plan of Action”, en Fu-Lai Tony Yu y Diana S. Kwan (comps.), Contemporary lssues in lnternational Political Economy, Singapur, Palgrave Macmillan, 2019.

Price, Richard, "Syria and the Chemical Weapons Taboo", Journal of Global Security Studies 4 (1), 2019, pp. 37-52.

Rотн, Kenneth, "Why the UN Chief's Silence on Human Rights Is Deeply Troubling”, The New York Times, 24 de abril de 2019.

Stromseth, Jane E., "An Imperial Security Council? Implementing Security Council Resolutions 1373 and 1390”, American Society of International Law Procedure, 97, 2003, p. 43.

"Ten Humanitarian Crises and Trends to Watch in 2019", The New Humanitarian, 2 de enero de 2019.

US Agency for International Development, "Climate Change Risk Profile: The Sahel”, 13 de abril de 2017. 
World Justice Project, "Rule of Law Index: 2017-2018”, 2018, https:/ / worldjusticeproject.org/our-work/research-and-data/wjp-rulelaw-index-2017\%E2\%80\%932018

Zorthian, Julia, "5 United Nations Achievements Worth Celebrating", Time, 23 de octubre de 2015, https:/ / time.com/4085757/ united-nations-achievements/ 